\title{
A Low Power AC/DC Interface for Wind-Powered Sensor Nodes
}

\author{
Mohammad Haidar ${ }^{1,2, * \mathbb{D}}$, Hussein Chible ${ }^{2}$, Corrado Boragno $^{3}$ (D) and Daniele D. Caviglia ${ }^{1}$ (D) \\ 1 Cosmic Lab, DITEN, University of Genoa, 16145 Genoa, Italy; daniele.caviglia@unige.it \\ 2 MECRL Lab, EDST, Lebanese University, BadaroBeirut P.O. Box 6573/14, Lebanon; hchible@ul.edu.lb \\ 3 Department of Physics (DIFI), University of Genoa, 16146 Genoa, Italy; corrado.boragno@unige.it \\ * Correspondence: mohammadhaidar555@gmail.com
}

check for updates

Citation: Haidar, M.; Chible, H.;

Boragno, C.; Caviglia, D.D. A Low

Power AC/DC Interface for

Wind-Powered Sensor Nodes.

Energies 2021, 14, 1823. https://

doi.org/10.3390/en14071823

Academic Editors: Noel Rodriguez and Rodolfo Araneo

Received: 17 December 2020

Accepted: 22 March 2021

Published: 25 March 2021

Publisher's Note: MDPI stays neutral with regard to jurisdictional claims in published maps and institutional affiliations.

Copyright: (C) 2021 by the authors Licensee MDPI, Basel, Switzerland. This article is an open access article distributed under the terms and conditions of the Creative Commons Attribution (CC BY) license (https:// creativecommons.org/licenses/by/ $4.0 /)$.

\begin{abstract}
Sensor nodes have been assigned a lot of tasks in a connected environment that is growing rapidly. The power supply remains a challenge that is not answered convincingly. Energy harvesting is an emerging solution that is being studied to integrate in low power applications such as internet of things (IoT) and wireless sensor networks (WSN). In this work an interface circuit for a novel fluttering wind energy harvester is presented. The system consists of a switching converter controlled by a low power microcontroller. Optimization techniques on the hardware and software level have been implemented, and a prototype is developed for testing. Experiments have been done with generated input signals resulting in up to $67 \%$ efficiency for a constant voltage input. Other experiments were conducted in a wind tunnel that showed a transient output that is compatible with the target applications.
\end{abstract}

Keywords: energy harvesting; switching converter; optimization; wind energy; maximum power point tracking (MPPT)

\section{Introduction}

The internet of things (IoT) is a hot topic that is evolving daily with the advancing technology, and the recent crisis raised interest and awareness in remote platforms and automotive tasks, giving the IoT an edge and pushing further towards more integration in this world. Numerous applications and fields are recently pivoting towards a common umbrella in IoT. Wireless sensor networks (WSN), automotive vehicles, smart homes and cities are all applications that define the future paired with the big investments in networking and next generation communication systems such as 5G.

Although data collection and communication by itself is not as challenging nowadays, especially in connected objects where data are limited in size and sparse in transmission time, the biggest challenge remains in power consumption and computational complexity.

As the connected objects are physically constrained in size and deployment location, reliable power sourcing becomes challenging. In fact, solutions that are based on battery charging are not reliable for many applications due to the high cost and effort of charging and changing the batteries periodically, mainly in applications where a huge number of nodes is present, or where the nodes are deployed in wild, random, or hard to reach locations. A good solution for this problem would be using energy harvesters to generate electrical energy from different energy sources present in nature. The main advantage is that the energy is unlimited in nature and can be present in different forms. When designed properly, and taking into consideration the spatial constrains, the energy harvesters can provide a long-term power source that is reliable and automatic, without the need for any human interaction.

Some challenges are present as the energy sources can also be a limited due to the fact that the environmental changes are random, and their availability could vary from one place to another (irradiation change, wind speed, etc.)

Historically, the energy of nature was made use of to perform important tasks, like agriculture and transportation. Nowadays many techniques have been developed to make 
use of the green energy such as wind turbines [1,2], solar panels [3,4], thermal energy harvesters [5] and even hybrid systems [6,7]. These harvester devices are used to transform energy from the source into an electrical form; and each harvester produces a different electrical signal with characteristics depending on its nature. This signal in its turn needs to be conditioned in order to be used in various applications that require a power source.

Some criteria need to be satisfied for the generated signal to useful: it needs to be regulated to the needed voltage level, stabilized, and be able to sustain certain load of the application of interest.

Energy harvesting can be separated to two types, high power and low power. The margin between them is defined by the point where the generated power reaches the same order of magnitude of the power consumed by the conditioning circuit.

High power harvesters such as wind turbines and large PV cells have more room for control and optimization, due to the fact that no hard constraints in size, weight or complexity are crucial to respect. On the other hand, low power harvesters are constrained by these factors, shifting the priority from optimizing and control into ensuring that the power is transferred and delivered to the output. The circuit has to be supplied from the source and maintain its supply at all cost.

The scope of this work is to design an electronic system to condition the signal generated by the novel wind harvester named Fluttering Energy Harvester for Autonomous Powering (FLEHAP) shown in Figure 1 [8]. This harvester is based on the fluttering instability effect as an alternative method to harvest energy from low wind speeds. It consists of a rigid wing that can freely rotate around an axis connected to the middle of tow elastomers, creating a self-sustained excitation of a two-degrees-of-freedom.

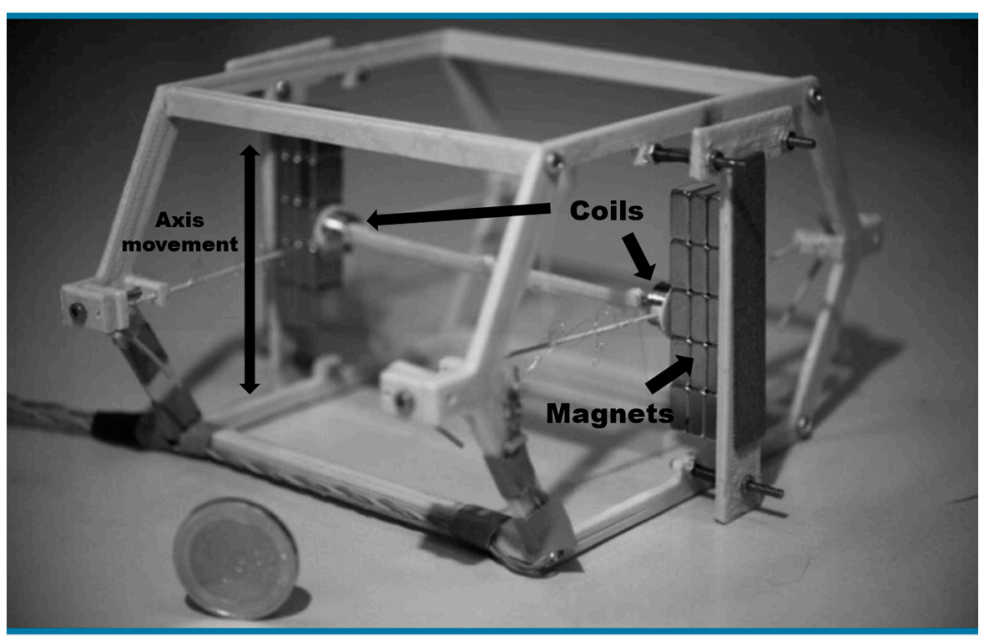

Figure 1. The wind energy harvester Fluttering Energy Harvester for Autonomous Powering (FLEHAP) [8].

Locally, the variations in wind speed over time can be considered of a random nature. Since such variations induce electromechanical changes to the operation of the harvester, in order to obtain the maximum possible power, it is necessary to adapt the behavior of the electronic circuit so that it can suitably follow the variations induced on the system by environmental variations.

Switching converters are often used to perform this task, as their equivalent input resistance (i.e., the impedance they present at the harvester output) depends on the duty cycle of the switching signal and can be controlled to automatically match the optimal value of the output resistance of the harvester.

Different algorithms are often used and many researchers suggest various ways to implement such algorithms, as well as their variations, using customized circuits [1-10], or by programming a microcontroller [11]. However, the freedom of choice among such 
algorithms is not allowed in low-power systems, due to the inherent trade-off between computational complexity and power consumption.

While such objectives generally raise some challenges, concerning the search for energy sources capable of satisfying energy needs and guaranteeing the correct functionality of the control circuits, further challenges in our case derive from the nature of the harvester itself and, consequently, from the shape of the signal it generates under different operating conditions, which entails some difficulties in monitoring the available power in real time and in optimizing its exploitation.

In our work, the main focus was performing the optimization considering both hardware and software, in contrast with other systems that only optimize through software $[12,13]$.

\section{Methodology}

The main goal of the energy harvesting system is to provide sufficient power to a given sensor node, so that it is able to perform its task normally. This sufficient power depends on the node itself, its radio communication protocol and the scheduling of its operations. A voltage regulation at the target level needs to be applied, while sustaining all the expected variations in the target load current and in the wind speed sweeping the harvester.

This goal requires the system to perform two tasks simultaneously:

1. Ensure and optimize the power transfer from the harvester.

2. Regulate and control the output supply current to satisfy the needs of the target node.

The block diagram in Figure 2 describes the function of different stages of the signal flow between them.

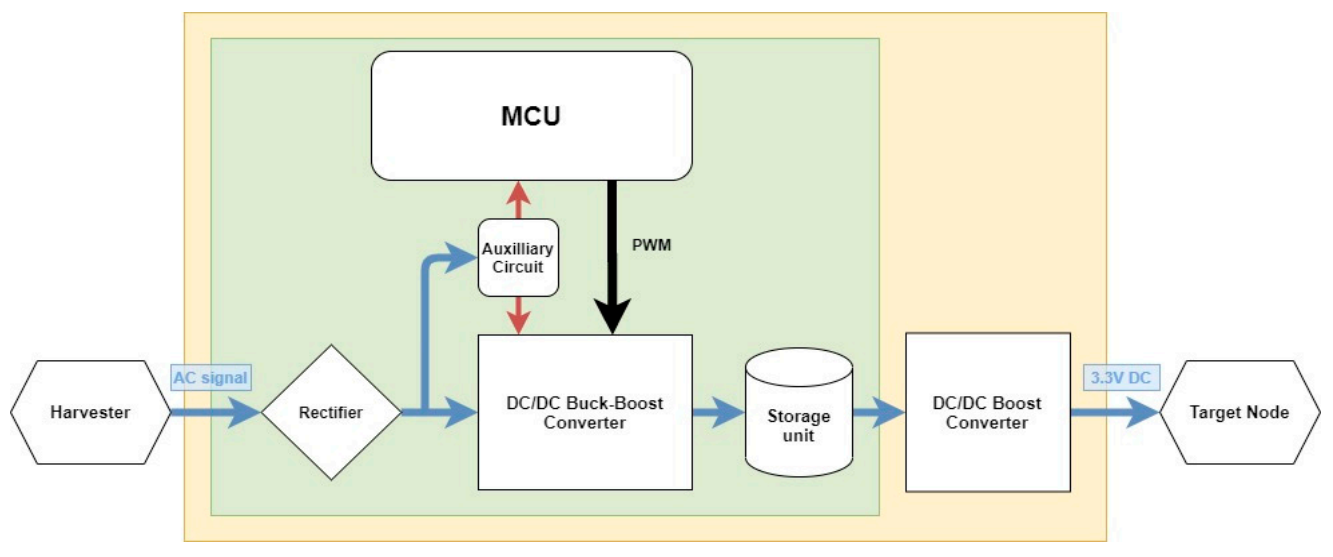

Figure 2. Block diagram of the proposed system.

A low power microcontroller is used here for three purposes. The first is to deliver a Pulse Width Modulated (PWM) signal to the DC/DC converter [14]. The second is to monitor the input power and run a maximum power point tracking (MPPT) algorithm for optimization. The third is to communicate the power state with an external device via serial communication $[15,16]$.

The input signal is split into two paths after being rectified. The auxiliary circuit consumes a small part of the power that can vary depending on the consumption of the microcontroller and the different active components used in the circuit. The rest of the power goes into the main DC/DC buck-boost converter that has the main purpose of dynamically adapting the input resistance to compensate for the weather conditions variation. The output energy is stored in the supercapacitor and then regulated to the required level using a second switching DC/DC converter. The choice of switching converters was made due to the fact that they provide high efficiency compared to passive regulators [17-19]. 
The green box is targeted in this work, while the second DC/DC boost converter is a conventional voltage regulator on a separate integrated circuit (IC) with the control mechanisms integrated.

DC/DC Buck-Boost converter: This type of converter uses a switching mechanism to charge an output capacitor. The topology in Figure 3 presents a non-inverting buck-boost DC/DC converter using three MOS transistors (MOSFETs) as switches. A PWM signal is applied to the switches to alternate between two electrical loops (S1 and S3 are in phase and S2 is in reverse phase with the PWM signal), the input loop that increases the inductor current and the output loop where the inductor plays the role of a current source that charges the super capacitor. The duty cycle of the PWM decides the ratio of the input to output voltage.

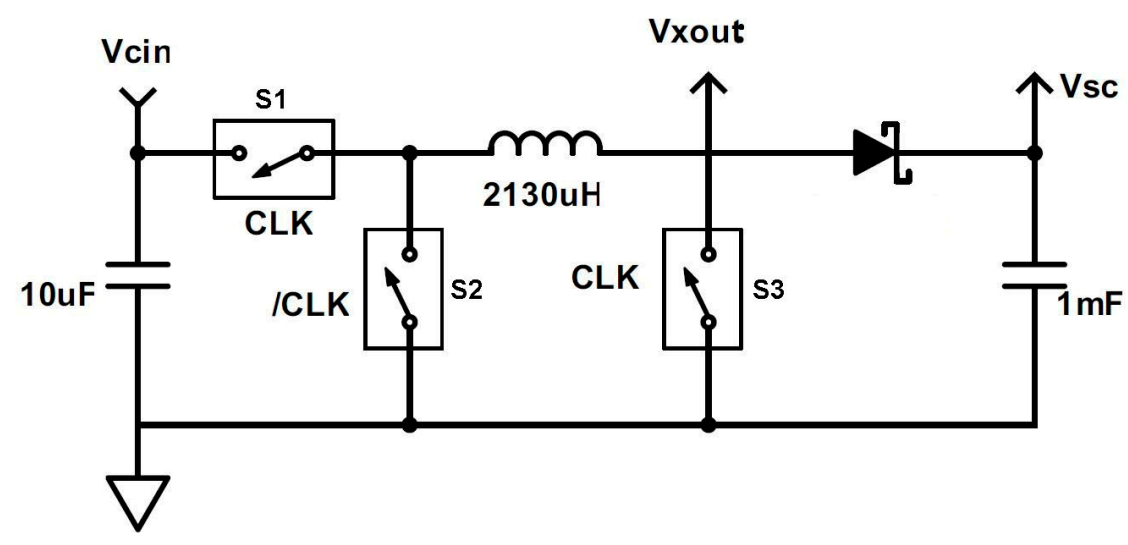

Figure 3. The used DC/DC converter schematic. Vcin, Vxout, Vsc refer to the input capacitor voltage, Anode voltage of the diode and super capacitor voltage, respectively.

Auxiliary circuit: This part of the system is responsible of powering all the active components in the circuit, including the Micro Controller Unit (MCU) and MOSFETs. The rectified input signal is regulated passively to deliver a stable DC voltage as shown in Figure 4. Although passive regulators cannot provide a high efficiency, it is necessary for the functioning of the system. For this reason, the load on the auxiliary circuit must be as low as possible.

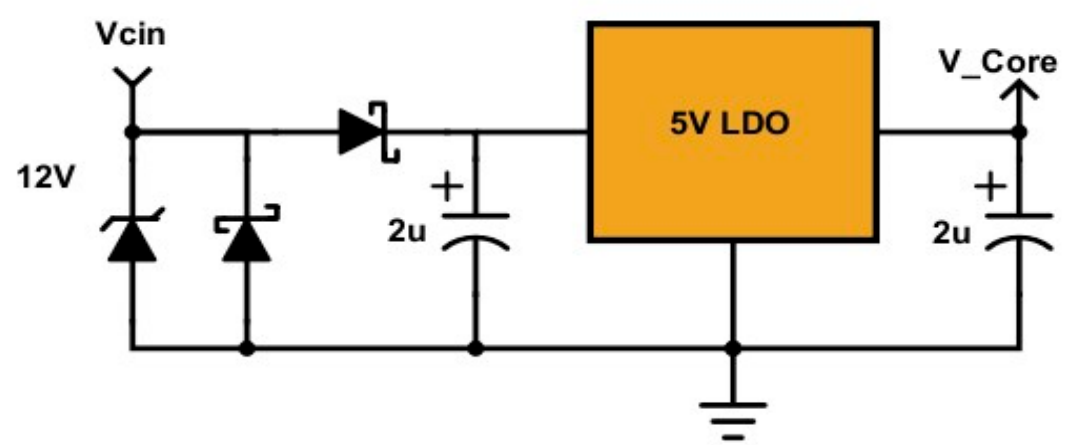

Figure 4. The auxiliary circuit used to provide a source (Vcore) for active components.

Super capacitor: The super capacitor is a more flexible storage unit than batteries, with a main drawback that is leakage current. In this system, the output current is not sufficient to charge commercial batteries like Li-Ion, and therefore a supercapacitor can fit here. With the correct configuration the supercapacitor can charge up during a fixed time period, which is the idle period for the sensor node, to store sufficient energy that can be delivered to the node during its active period. 


\section{Power Optimization}

Power optimization is crucial in low power energy harvesting systems, and the gained advantages are what makes such systems useful and competing in the power supply field.

The optimization must be done either on the hardware where the loss in different hardware components should be minimized while maintaining a good performance, or it could be done on the software level where different algorithms kick in to minimize the power consumption by the computations and to dynamically match the circuit impedance for a better power transfer using MPPT algorithms.

\subsection{Hardware Optimization}

Power loss occurs in all components of the circuit, and there are no ideal components that one can make use of in practice. However, some conventional electronic parts can be substituted with small circuits that keep the same functionality while consuming less power. The diodes have been always perceived as a source of power loss and have been actively substituted in low power circuits. A mathematical model based on equations from [8] was developed and simulated in MATLAB to simulate the power consumption of different parts in the converter circuit with only the switches, coil, diode and output capacitor. The following equations represent the average power dissipated in the diode and switches and the output energy stored in the capacitor during one PWM cycle. $I_{p, k}$ is the peak inductor current during the cycle $k, R_{s w}$ is the switches ON resistance, $V_{L, k}$ is the inductor voltage and $V_{\text {out }, k}$ is the voltage across the capacitor.

$$
\left\{\begin{array}{c}
P_{D, k}=\frac{I_{P, k}}{2} V_{T} \ln \left(1+\frac{I_{P, k}}{2 I_{s}}\right) \\
P_{s w, k}=R_{s w}\left(\frac{I_{P, k}}{2}\right)^{2} \\
E_{\text {out }, k}=\frac{C}{2}\left(V_{\text {out }, k}\right)^{2}=\frac{C}{2}\left(\frac{L I_{p, k}^{2}}{2 C V_{L, k}}+V_{\text {out }, k-1}\right)^{2}
\end{array}\right.
$$

Figure 5 shows that the diode in the output loop of the DC/DC buck-boost converter consumes a relatively high amount of power that is supposed to reach the super capacitor, especially when the output voltage is low.

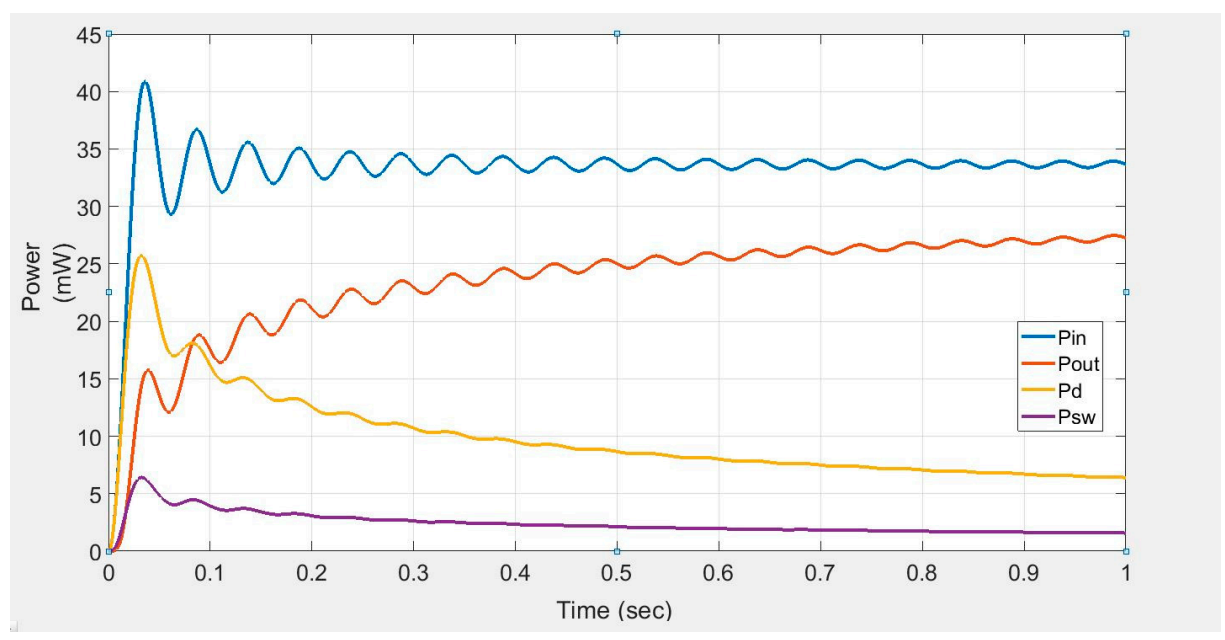

Figure 5. Simulation showing the consumed power in the different circuit components. The values Pin, Pout, Pd, Psw refer to the input power, output power, power consumed by the diode and power consumed by the switches, respectively.

In previous works where hardware optimization had proven useful, is it suggested to replace the diodes in the bridge rectifier with $\mathrm{N}$ and $\mathrm{P}$ channel MOSFETs driven by the alternate (AC) input signal [20]. A similar approach is taken in this system to replace all 
diodes with MOSFETs. For the diode in the output loop, a MOSFET is used with a smart diode driver integrated circuit, to prevent reverse polarity current from flowing in the super capacitor and therefore discharging it on every cycle.

\subsection{Input Matching}

In this article, the term software optimization is used to describe the optimization algorithm that is ran by the software in the MCU on the conditioning system, and not the power optimization on the host node.

A common issue in energy harvesters is that some characteristics vary with the change of weather conditions. Due to the inductive nature of the harvester, a reverse induced force applies a brake effect on the wing whenever current runs through the coil. To generate more voltage, the current must be decreased resulting in a lower power generated. Thus, a tradeoff between the generated voltage and current exists and needs to be controlled to find the optimal power transfer point.

The graph in Figure 6 shows the variation of maximum generated power in respect to the load resistance, and it shows that the characteristics and the maximum power point changes with the variation of wind speed. The graph is an interpolation of the data collected by the experiment in [21], where the dots are the experimental values, and the lines are a Gaussian interpolation; different curves represent different wind speeds.

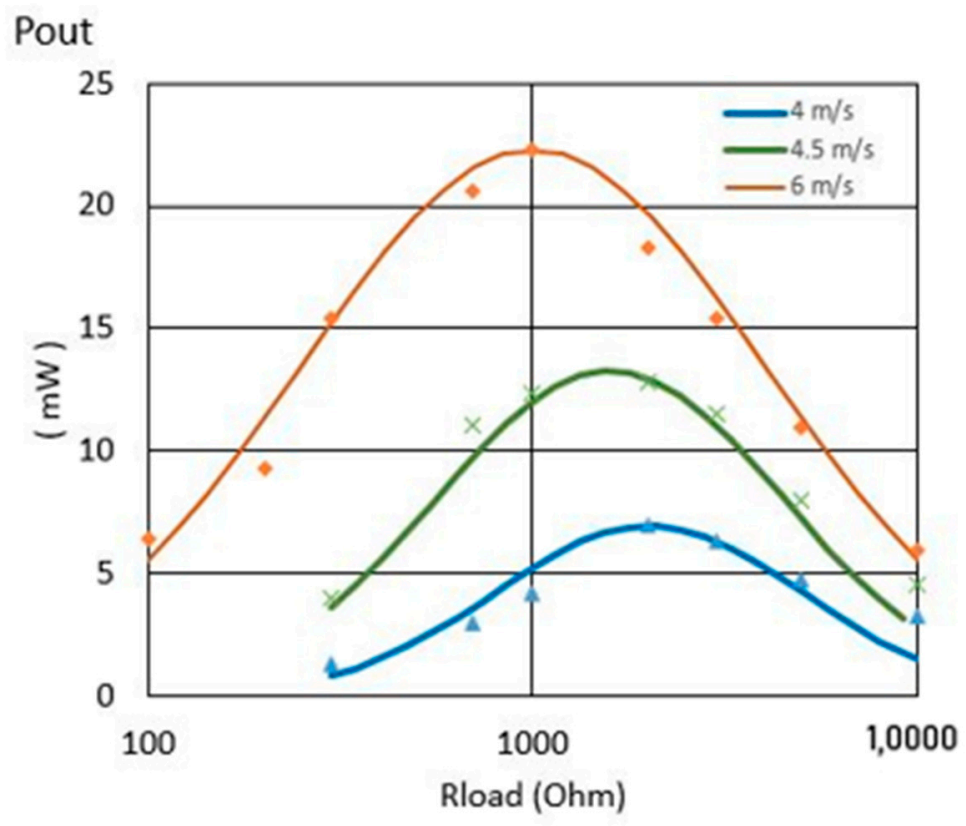

Figure 6. Available power from the FLEHAP harvester depending on the wind speed and the load resistance.

The choice of the DC/DC buck-boost converter is due to the ability to control its equivalent input resistance by changing the PWM duty cycle. In DCM mode, the input resistance can be independent of the load on the output of the DC/DC converter, and it can be expressed with the following equation [8]:

$$
R_{E Q}=\frac{2 L_{H}}{D^{2} t_{C K}}
$$

where $L_{H}$ is the harvester's inductance, and $D$ and $t_{C K}$ the duty cycle and the period of the PWM clock signal, respectively. The MCU controls $D$, and consequently $R_{E Q}$, using a MPPT algorithm to adapt the equivalent input resistance, where the goal is to find the optimal power point following the modifications the overall system undergoes due to wind speed changes. 


\section{Algorithm}

Energy harvesting systems have been relying on MPPT algorithms to control many factors leading to power optimization. Depending on the nature of the system, the algorithms can change and become more or less complex. Many researchers suggested solutions and algorithms for the maximum power point tracking (MPPT) such as Hill Climb Search (HCS) algorithms including:

- Perturb and Observe (P\&O) $[4,22,23]$. This algorithm works by introducing small perturbations in a control variable, and adapting these perturbations depending on the direction of the monitored power.

- Incremental Conductance (INC) [24]. The principle of this algorithm is defined by the relation:

$$
\frac{i}{v}+\frac{d i}{d v}=0
$$

This tangent slope should be zero on the MPP, while positive on the left, and negative on the right of such a point.

- Lookup tables and pre-obtained models [2]. This method simply works by doing experiments on the system before-hand, and deducing the maximum power transfer point of the system by monitoring some parameters and searching the lookup table.

- Fuzzy logic [25]. This algorithm is defined for wind turbines and takes multiple parameters then calculates the output control parameters by using the rule base and defuzzification method.

- Neural networks [26]. The neural network is trained on a dataset that is collected from experiments, consisting of input data being the different test points and the output data being the control variables.

The possible computational complexity of such algorithms is not a concern when they are intended to operate on large plants, but it becomes a serious problem in low-power systems, when the harvested power may be in the order of magnitude of that consumed by the control circuits.

Consequently, we have decided to adopt the $\mathrm{P} \& \mathrm{O}$ algorithm, which is one of the simplest and consumes less energy.

\subsection{The Proposed $P \mathcal{E} O$}

The $\mathrm{P} \& \mathrm{O}$ method is one of the simplest methods used for the maximum power tracking. The advantages it offers are low computational requirements and low cost due to the independence on mechanical sensors and measurements. The input resistance of the switching converter follows the switching duty cycle, and by applying small perturbations to it, the received power changes and therefore it could be controlled to reach the MPP.

In the new modified $\mathrm{P} \& \mathrm{O}$ algorithms, an adaptive duty cycle step that is proportional to the power gradient is usually used instead of a fixed step, to speed up the convergence and to get rid of the ripples around the maximum point.

However, due to the lack of accuracy and relatively high noise in the voltage measurement, the modified MPPT algorithm seems to be highly inconsistent and mislead by the slightest of perturbations in this application. Therefore, the $\mathrm{P} \& \mathrm{O}$ algorithm that is shown in Figure 7 is proposed. The new algorithm uses a fixed step duty cycle and takes the case where $\mathrm{dP}$ is less than a specified threshold as a stop condition, to prevent losses caused by the ripples around the MPP. 


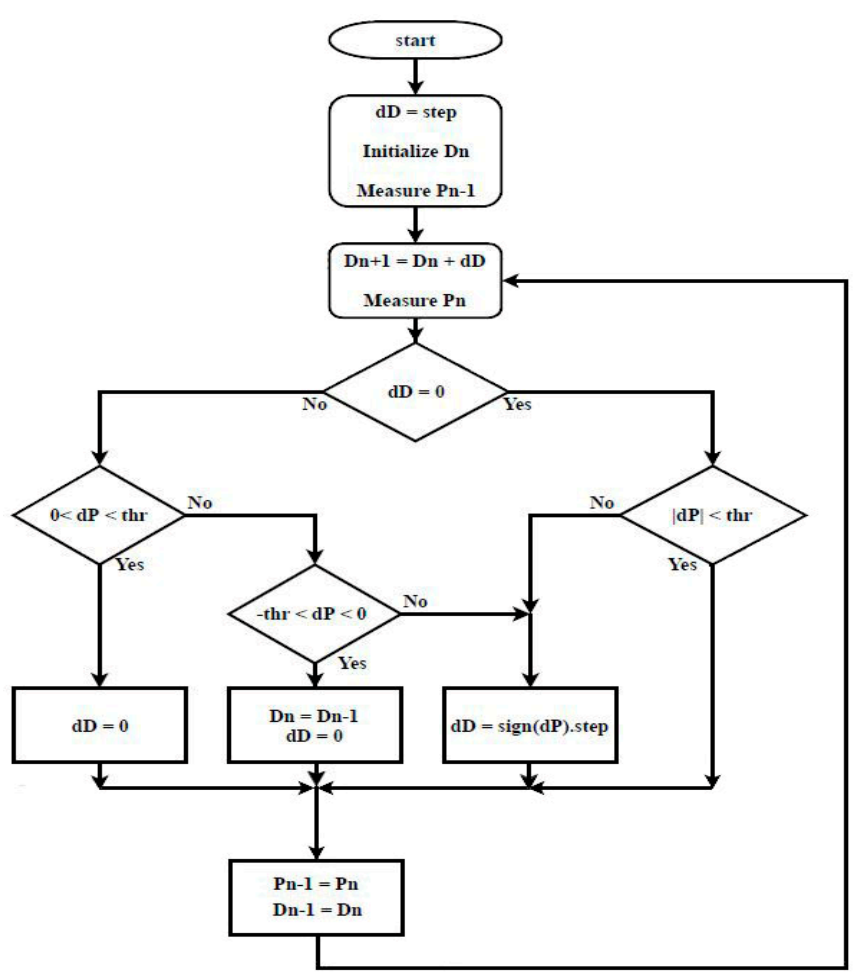

Figure 7. The proposed Perturb and Observe (P\&O) algorithm.

The condition where the power differential is under threshold but on the downside of the power slope is considered separately $(-\mathrm{thr}<\mathrm{dP}<0)$, where the duty cycle is set to the previous value before skipping the maximum power point downwards. $D_{n}$ and $D_{n-1}$ are the new and old duty cycle, $P_{n}$ and $P_{n-1}$ the respective measured power and $\mathrm{dP}=\mathrm{P}_{\mathrm{n}}-\mathrm{P}_{\mathrm{n}-1}$.

An experiment was conducted to emulate the effect of changing wind speed, by using a computer-generated voltage signal, and changing the series resistance of the input, forcing the algorithm to adapt and follow the maximum power. The two algorithms were used in this experiment. Figure 8 shows the difference in the performance of the MPPT using the variable step algorithm and the new proposed one.

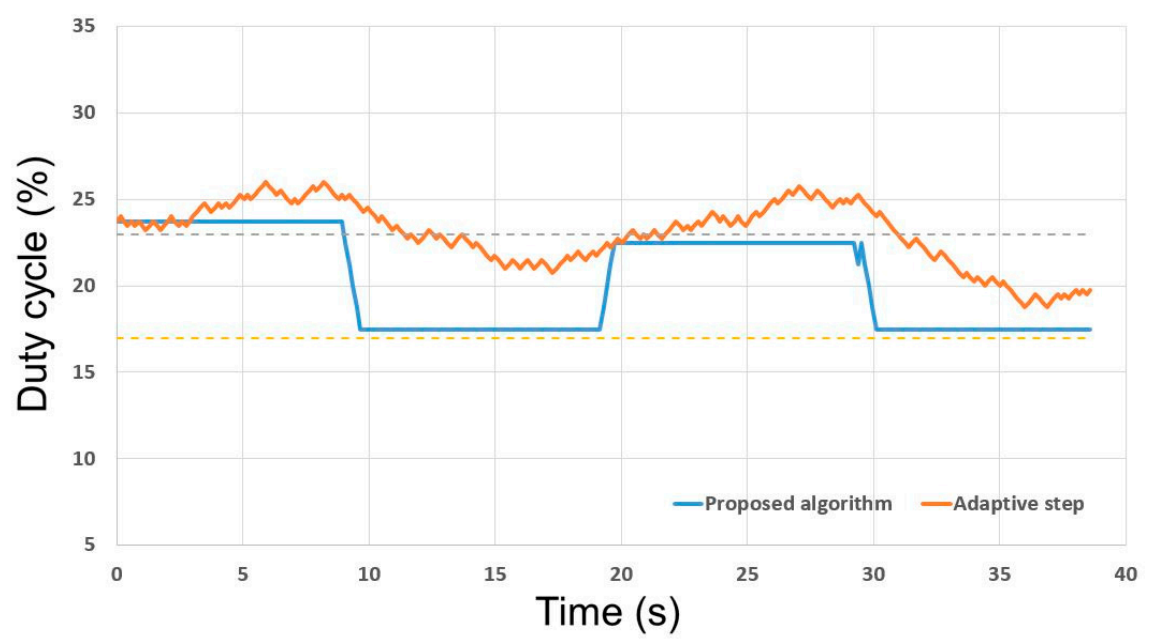

Figure 8. Experiment showing the difference in response between the $\mathrm{P} \& \mathrm{O}$ algorithm with adaptive step (orange), and the proposed algorithm (blue). 
An error on the equivalent resistance is present around the MPP due to the fixed step (sampling error). This error can be calculated depending on the step size and the duty cycle by

$$
\varepsilon_{D}(m)=\frac{\Delta R(m)}{R}=1-\left(\frac{D}{D+m}\right)^{2}
$$

where $m$ is the step size and $D$ is the duty cycle.

This relationship can be visualized in Figure 9; the error increases with step size and with the resistance value we want to emulate.

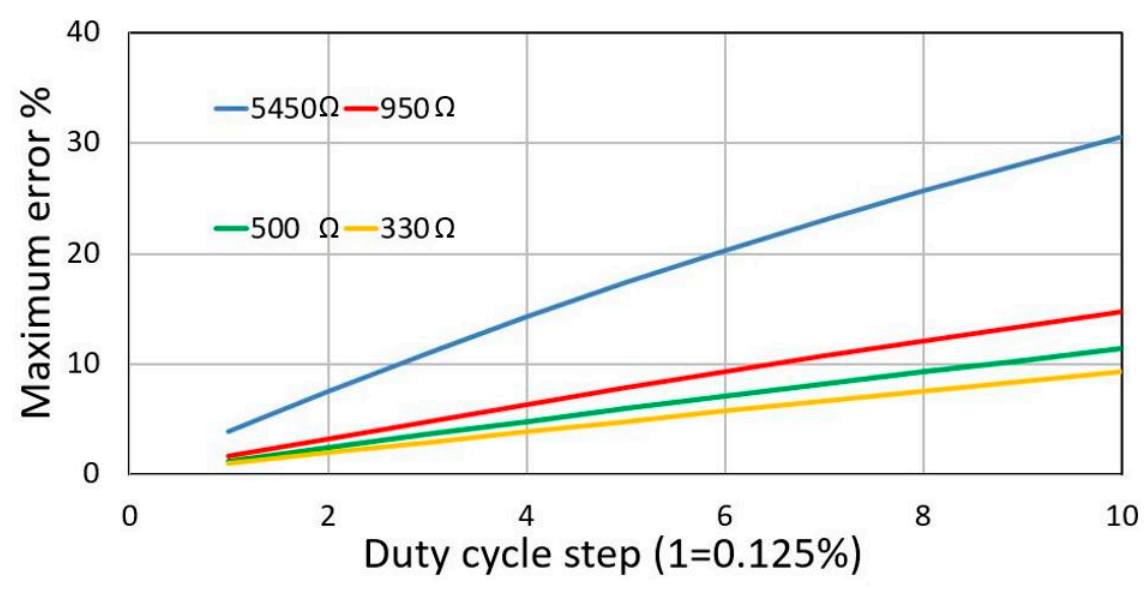

Figure 9. Plot showing the relative maximum error with respect to the fixed step size of the duty cycle. The different plots represent different equivalent resistance values.

In the previous experiment on the FLEHAP harvester [19], the $\mathrm{P}(\mathrm{R})$ characteristic in Figure 6 shows that, as the wind speed decreases, the curve becomes flatter, the maximum widens, and consequently the resistance margin associated with a fixed error $\Delta \mathrm{P}$ around the maximum increases as well. Therefore, higher errors on the equivalent resistance value, with respect to the optimal one, can be tolerated. Thus, the step size can be fixed in a way to achieve a bounded error on the input power Pin.

\subsection{Voltage Reading}

In the prototype used in this work, the output voltage of the harvester presents a fundamental frequency in the range of $10 \mathrm{~Hz}$, with significant harmonics up to the fifth. Higher frequencies are also present, because of the switching mechanism in the DC/DC converter which operates at $5 \mathrm{kHz}$ (Figure 10). All these facts induce possible errors (noise) in measuring such voltage. For consistent voltage reading, each analog to digital conversion (ADC) acquisition needs to be synchronized with the voltage ripples. The high frequency synchronization can be done by using the falling edge of the PWM as an interrupt signal, and acquiring the voltage inside the interrupt routine. This approach will limit the algorithm update speed to the period of the input signal. The algorithm tracks the variation of power and thus the instant value is not as important as the differential value. For this reason, it is sufficient to measure the peak $\mathrm{AC}$ voltage and rely on it to monitor the variation of power. 


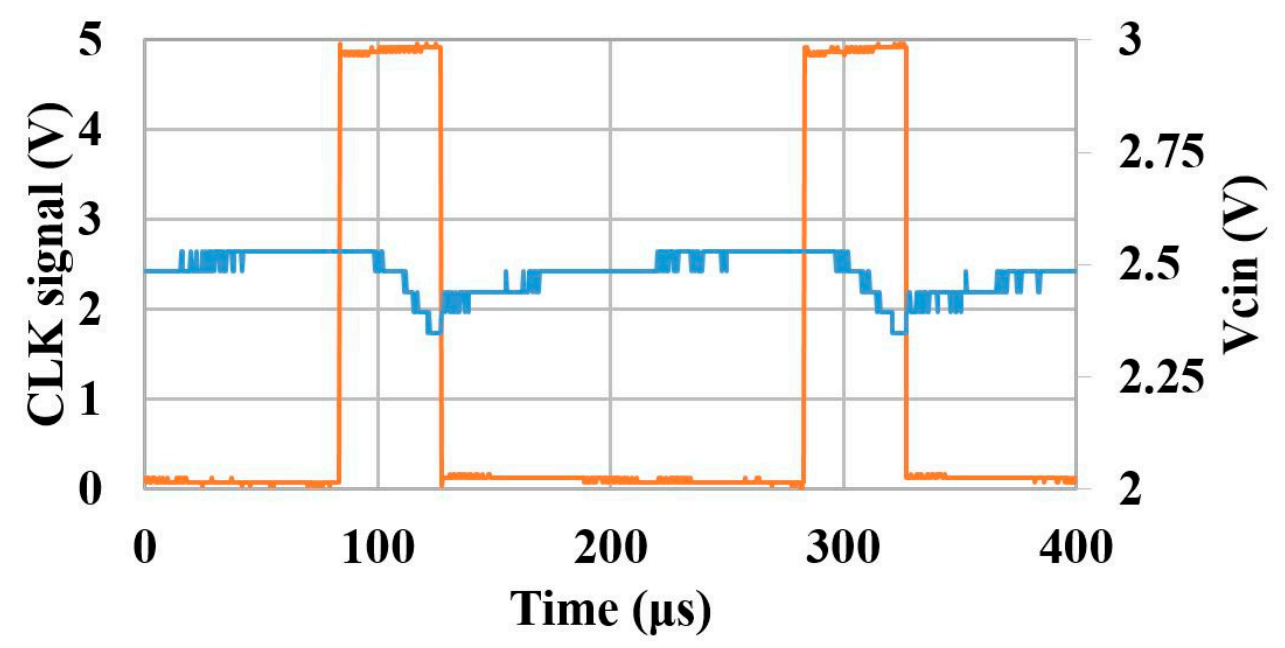

Figure 10. Plot showing the high frequency ripples in synchronization with the PWM pulses.

The maximum input power provided by this system is in the order of magnitude of a few tens of milliwatts, when the wind speed reaches around $5 \mathrm{~m} / \mathrm{s}$ [8]. If the wind speed decreases, the extracted power can become insufficient to meet the needs of both active electronics and software calculations.

In this critical case, the system may need to sacrifice the optimal input power, and maintain a sufficient voltage for the circuit operation by decreasing the input current. For this purpose, two thresholds are set, namely Vth_min and Vth_max, thus establishing a hysteretic behavior. In this way the system enters the critical state whenever Vcore $<$ Vth_min, and leaves the critical state when Vcore $>V t h \_$max.

During the critical state, the MPPT algorithm is disabled, and the PWM is set to $D_{M I N}$, the lowest duty cycle possible that remains detectable by the external interrupt of the MCU. This mode of operation produces the highest equivalent resistance on the input. The optimization state is the normal operation state where the MPPT algorithm is running, starting from an initial condition with $D=D_{M I N}$.

\section{Experiments}

For the sake of measuring the efficiency of the system, a PCB was manufactured containing the buck-boost converter, the MCU and the auxiliary circuit.

The used design was a non-inverting buck-boost converter containing three lowresistance MOSFET switches ADG801 from Analog Devices and a $2.3 \mathrm{mH}$ inductor. On the input, a MOSFET full wave rectifier was used, for the reason that it showed an improvement in the efficiency over a Schottcky bridge rectifier in LTSpice simulation (an increase from $73 \%$ to $88 \%$ ). The output diode was replaced with an N-channel MOSFET controlled by a Texas Instruments LM74810Q smart diode controller. A $C_{\text {STORE }}$ electrolytic capacitor of $1 \mathrm{mF}$ was placed on the output as an energy storage unit. This value was used in the prototype as a proof of concept while in different cases the values of the supercapacitor is calculated depending on the application's energy need. This experiment used a pic16F1788 from Microchip as a microcontroller to run the MPPT algorithm.

- DC Input

This experiment was done using a voltage source that has a $330 \Omega$ series resistance. This value is the same as the series resistance of the "FLEHAP" harvester under maximum wind speed. The PWM duty cycle was fixed to give a matching input and a load of $120 \Omega$, connected in parallel with $C_{\text {STORE. }}$. Figure 11a shows the Vcin and Vout at the transient phase for a $V_{S}=6 \mathrm{~V}$ input, resulting in an output power of $18.25 \mathrm{~mW}$ and an efficiency of $67 \%$ after $0.2 \mathrm{~s}$. The MCU here consumed $14.7 \%$ of the input power and this was measured through a small series resistor in the circuit with $1 \Omega$ resistance. The power consumption 
of the MCU was measured in the circuit without performing any calculation regarding computational operations.

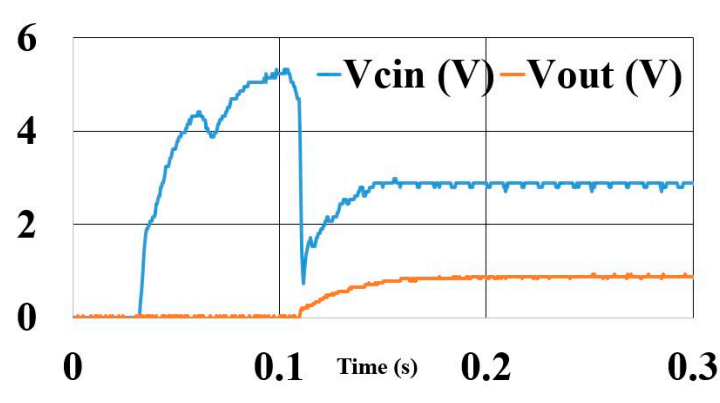

(a)

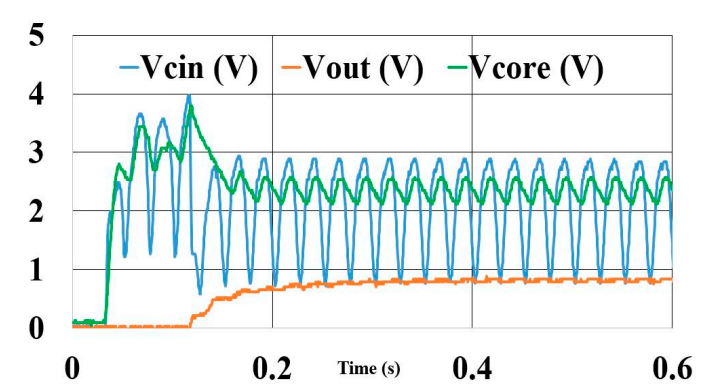

(b)

Figure 11. Transient of the system with (a) DC input and (b) AC input where Vcore is the output of the auxiliary circuit.

We calculated the efficiency using the following equation, where $R_{\mathrm{S}}$ is the input series resistance:

$$
\text { eff }=\frac{P_{\text {out }}}{P_{\text {in, } \max }}=\frac{P_{\text {out }}}{\frac{V_{\text {cin, } \max }^{2}}{R_{s}}}=\frac{18.25}{27.27}=0.67
$$

- $\quad$ AC Input

An input $20 \mathrm{~Hz}$ sine wave with $6 \mathrm{~V} \mathrm{Vp}$ provided enough voltage at Vcore to power up the MCU and run the algorithm as shown in Figure 11b. The output power it could provide was around $5.824 \mathrm{~mW}$, giving an efficiency of $54.7 \%$.

\section{- Wind Tunnel Experiment}

Several experiments were conducted on the whole system including the real FLEHAP harvester and using a DC/DC boost converter based on a Monolithic Power Systems (MPS) MP28164 voltage regulator to obtain $3.3 \mathrm{~V}$ at the output. The experiments were made in a controllable wind tunnel (Figure 12) that can be operated at different wind speed.

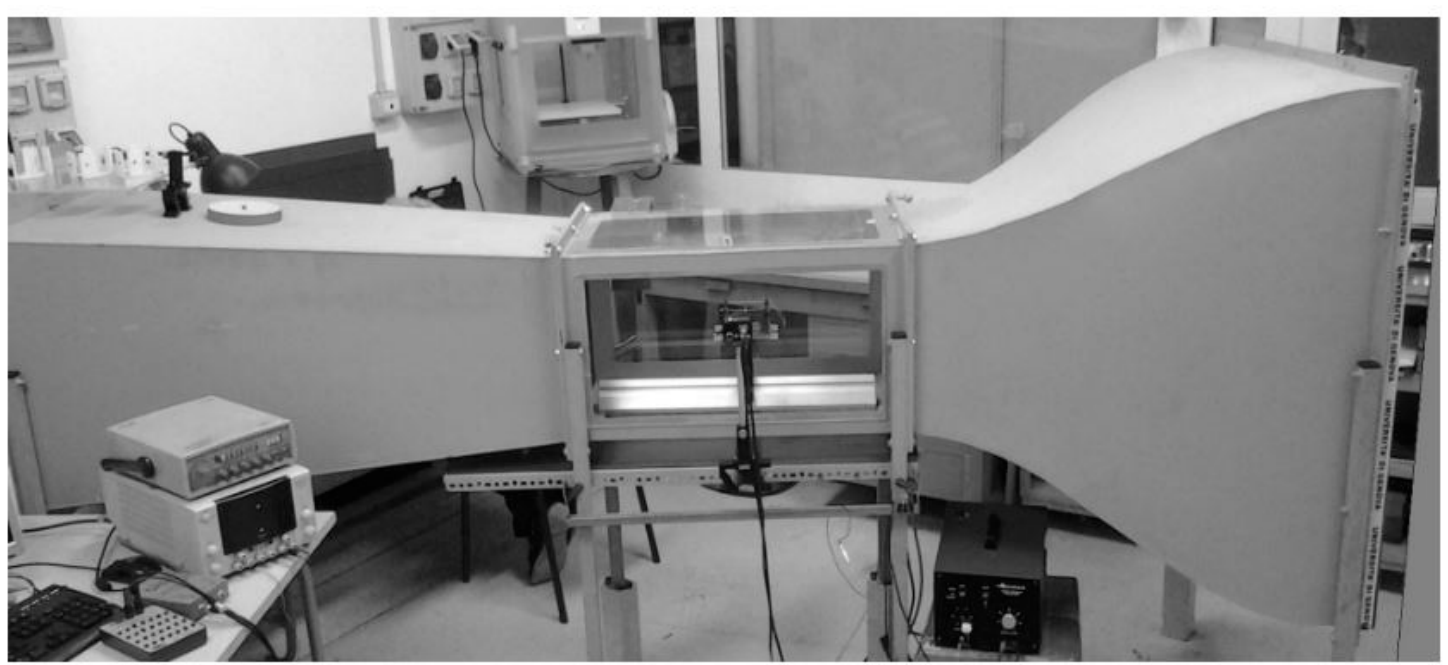

Figure 12. The wind tunnel used for the experiments.

Figure 13 shows the super capacitor voltage along with the output voltage of the system under a wind speed of $2.4 \mathrm{~m} / \mathrm{s}$. The circuit operation was initiated with a load of $22 \mathrm{~K} \Omega$ consuming $495 \mu \mathrm{W}$ and after the super capacitor stored enough energy to trigger 
the output converter, a load of $6 \mathrm{~K} \Omega$ was introduced, representing a power consumption of $1.815 \mathrm{~mW}$. The super capacitor discharged in around $2 \mathrm{~s}$ following the relation:

$$
C=I_{C} \frac{\Delta t}{\Delta V}
$$

where $I_{C}$ can be approximated from the rated efficiency of the DC/DC converter.

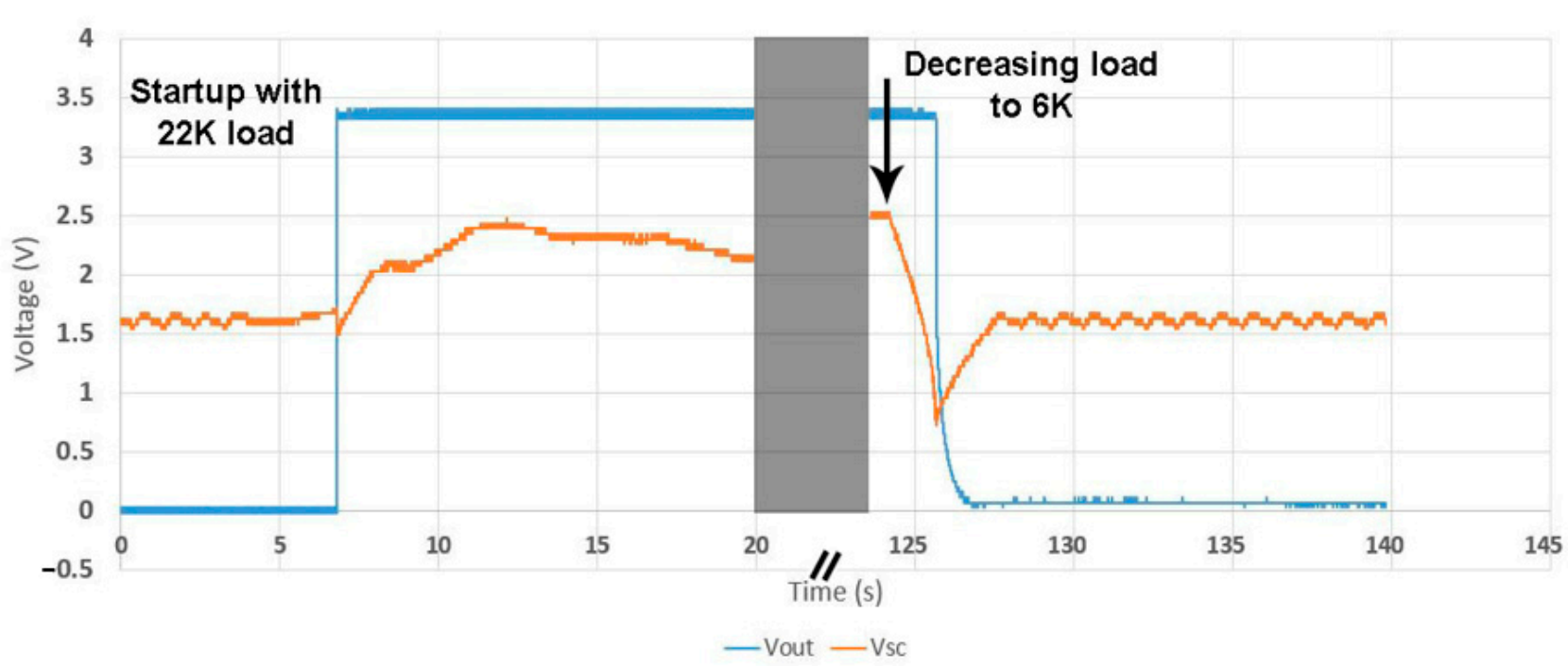

Figure 13. Plot showing the response of the system in typical conditions and under changing loads.

This experiment shows the mechanism on a small scale, while for typical application a correct super capacitor value must be calculated to sustain the needed current for the needed duration, the charging time can be also deduced respectively, and so on.

\section{Conclusions and Future Work}

This article presents an electronic interface for a novel fluttering wind energy harvester named FLEHAP. It can be described as a voltage conditioning system with the objective to power small wireless sensor nodes optimizing the power extraction from the environment and regulating its DC voltage at the output.

A detailed description of the system was presented, with an analytical study that allowed location of the source of power losses and its minimization. The system implements a technique called maximum power point tracking (MPPT) using a low power microcontroller on board, with a goal to match its input load with the harvester capabilities and optimize the power transfer. The MPPT algorithm is a standard Hill climb search algorithm that has been modified to fulfill the system requirements. Other software considerations were also mentioned that are essential to navigate the low wind speed conditions and thus the lack of sufficient input power, which often occurs due to the random environment.

A prototype of the system was produced and then tested in laboratory experiments, first with signal generators replacing the real harvester and producing different forms of signals mainly DC and AC sinusoidal signals. These laboratory tests were completed by experiments carried out inside a controllable wind tunnel with the real wind harvester. The results allowed estimation of the efficiency of the harvester, the ability to match the input using the MPPT algorithm, and the transient behavior that is useful to customize the system for different sensor nodes current requirements.

Future work aims to compensate for the downtime in the wind speed that results from a lack of input power, by the integration of another energy harvester (e.g., solar panel, temperature gradient, etc.) and therefore upgrading the system into a hybrid energy harvesting interface. 
Author Contributions: Conceptualization, M.H., C.B. and D.D.C.; Investigation, M.H.; Methodology, M.H., C.B. and D.D.C.; Project administration, D.D.C.; Software, M.H.; Supervision, H.C. and D.D.C.; Validation, D.D.C.; Writing—original draft, M.H.; Writing—review \& editing, M.H., H.C. and D.D.C.; Resources, C.B. All authors have read and agreed to the published version of the manuscript.

Funding: This research was funded in part by the Italian Ministry of Education, University and Research (MIUR), grant no. DM64549 “MAREA—Monitoring And Rescue Automation”.

Conflicts of Interest: The authors declare no conflict of interest.

\section{References}

1. Aubrée, R.; Auger, F.; Macé, M.; Loron, L. Design of an efficient small wind-energy conversion system with an adaptive sensorless MPPT strategy. Renew. Energy 2016, 86, 280-291. [CrossRef]

2. Wang, Q.; Chang, L. An Intelligent Maximum Power Extraction Algorithm for Inverter-Based Variable Speed Wind Turbine Systems. IEEE Trans. Power Electron. 2004, 19, 1242-1249. [CrossRef]

3. Kim, H.; Kim, S.; Kwon, C.-K.; Min, Y.-J.; Kim, C.; Kim, S.-W. An Energy-Efficient Fast Maximum Power Point Tracking Circuit in an 800- $\mu \mathrm{W}$ Photovoltaic Energy Harvester. IEEE Trans. Power Electron. 2013, 28, 2927-2935. [CrossRef]

4. Hussein, K. Maximum photovoltaic power tracking: An algorithm for rapidly changing atmospheric conditions. IEE Proc. Gener. Transm. Distrib. 1995, 142, 59. [CrossRef]

5. Toyama, Y.; Ogawa, T.; Ueno, T.; Itakura, T. $20 \mathrm{mV}$ input, $4.2 \mathrm{~V}$ output SIDO boost converter with low-power controller and adaptive switch size selector for thermoelectric energy harvesting. In Proceedings of the 2016 IEEE Asian Solid-State Circuits Conference (A-SSCC), Toyama, Japan, 7-9 November 2016; Volume 7, pp. 9-12.

6. Ambrosio, R.; Torrealba, R.; Guerrero-C., J.; Gonzalez, V.; Limon, A.; Moreno, M. Energy harvesting combining three different sources for low power applications. In Proceedings of the 2015 12th International Conference on Electrical Engineering, Computing Science and Automatic Control (CCE), Mexico City, Mexico, 26-30 October 2015; pp. 1-6.

7. Chamanian, S.; Çiftci, B.; Uluşan, H.; Muhtaroğlu, A.; Külah, H. Power-Efficient Hybrid Energy Harvesting System for Harnessing Ambient Vibrations. IEEE Trans. Circuits Syst. Regul. Pap. 2019, 66, 2784-2793. [CrossRef]

8. Boccalero, G.; Boragno, C.; Caviglia, D.D.; Morasso, R. FLEHAP: A Wind Powered Supply for Autonomous Sensor Nodes. J. Sens. Actuator Netw. 2016, 5, 15. [CrossRef]

9. Kumar, D.; Chatterjee, K. A review of conventional and advanced MPPT algorithms for wind energy systems. Renew. Sustain. Energy Rev. 2016, 55, 957-970. [CrossRef]

10. Chen, S.-H.; Huang, T.-C.; Ng, S.S.; Lin, K.-L.; Du, M.-J.; Kang, Y.-C.; Chen, K.-H.; Wey, C.-L.; Lin, Y.-H.; Lee, C.-C.; et al. A Direct AC-DC and DC-DC Cross-Source Energy Harvesting Circuit with Analog Iterating-Based MPPT Technique with 72.5\% Conversion Efficiency and 94.6\% Tracking Efficiency. IEEE Trans. Power Electron. 2015, 31, 5885-5899. [CrossRef]

11. Kumar, S.; Thakura, P. Microcontroller based DC-DC Cascode Buck-Boost Converter. In Proceedings of the 2017 Third International Conference on Advances in Electrical, Electronics, Information, Communication and Bio-Informatics (AEEICB), Chennai, India, 27-28 February 2017; pp. 289-294.

12. Janković, S.; Member, S.; Popović, I.; Lekić, A.; Saranovac, L. Power management for wireless sensor nodes. In Proceedings of the IcETRAN-2015, Silver Lake, Serbia, 8-11 June 2015; pp. 5-8.

13. Wu, Y.; Liu, W.; Zhu, Y. Design of a wind energy harvesting wireless sensor node. In Proceedings of the 2013 IEEE Third International Conference on Information Science and Technology (ICIST), Yangzhou, China, 23-25 March 2013; pp. 1494-1497. [CrossRef]

14. Marian, K. Kazimierczuk, Pulse-Width Modulated DC-DC Power Converters, 2nd ed.; Wiley: Hoboken, NJ, USA, 2015; ISBN 978-1-119-00954-2.

15. Haidar, M.; Chible, H.; Di Zitti, E.; Caviglia, D.D. An Optimized AC/DC Buck-Boost Converter for Wind Energy Harvesting Application. In Proceedings of the 2019 IEEE International Conference on Environment and Electrical Engineering and 2019 IEEE Industrial and Commercial Power Systems Europe (EEEIC/I\&CPS Europe), Genova, Italy, 11-14 June 2019; pp. 1-4. [CrossRef]

16. Haidar, M.; Chible, H.; Morasso, R.; Caviglia, D.D. AC/DC Buck Boost Converter for Wind-Powered Wireless Sensors. In Proceedings of the 2019 15th Conference on Ph.D Research in Microelectronics and Electronics (PRIME), Lausanne, Switzerland, 15-18 July 2019; pp. 41-44.

17. Saravanan, S.; Babu, N.R. Design and Development of Single Switch High Step-Up DC-DC Converter. IEEE J. Emerg. Sel. Top. Power Electron. 2017, 6, 855-863. [CrossRef]

18. Kruse, K.; Elbo, M.; Zhang, Z. GaN-based high efficiency bidirectional DC-DC converter with $10 \mathrm{MHz}$ switching frequency. In Proceedings of the 2017 IEEE Applied Power Electronics Conference and Exposition (APEC), Tampa, FL, USA, 26-30 March 2017; pp. 273-278.

19. Lai, C.-M.; Cheng, Y.-H.; Teh, J.; Lin, Y.-C. A New Combined Boost Converter with Improved Voltage Gain as a Battery-Powered Front-End Interface for Automotive Audio Amplifiers. Energies 2017, 10, 1128. [CrossRef]

20. Porcarelli, D.; Spenza, D.; Brunelli, D.; Cammarano, A.; Petrioli, C.; Benini, L. Adaptive Rectifier Driven by Power Intake Predictors for Wind Energy Harvesting Sensor Networks. IEEE J. Emerg. Sel. Top. Power Electron. 2014, 3, 471-482. [CrossRef] 
21. Boccalero, G.; Boragno, C.; Morasso, R.; Caviglia, D.D. A Sensor Node Driven by Air Flow. In Proceedings of the 2017 New Generation of CAS (NGCAS), Genova, Italy, 6-9 September 2017; pp. 21-24. [CrossRef]

22. Kazmi, S.M.R.; Goto, H.; Guo, H.-J.; Ichinokura, O. A Novel Algorithm for Fast and Efficient Speed-Sensorless Maximum Power Point Tracking in Wind Energy Conversion Systems. IEEE Trans. Ind. Electron. 2011, 58, 29-36. [CrossRef]

23. Femia, N.; Petrone, G.; Spagnuolo, G.; Vitelli, M. Optimization of Perturb and Observe Maximum Power Point Tracking Method. IEEE Trans. Power Electron. 2005, 20, 963-973. [CrossRef]

24. Hohm, D.; Ropp, M. Comparative study of maximum power point tracking algorithms using an experimental, programmable, maximum power point tracking test bed. In Proceedings of the Conference Record of the Twenty-Eighth IEEE Photovoltaic Specialists Conference-2000 (Cat. No.00CH37036), Anchorage, AK, USA, 15-22 September 2000; pp. 1699-1702. [CrossRef]

25. Algazar, M.M.; Al-Monier, H.; El-Halim, H.A.; Salem, M.E.E.K. Maximum power point tracking using fuzzy logic control. Int. J. Electr. Power Energy Syst. 2012, 39, 21-28. [CrossRef]

26. Li, H.; Shi, K.; McLaren, P. Neural network based sensorless maximum wind energy capture with compensated power coefficient. In Proceedings of the Conference Record of the 2004 IEEE Industry Applications Conference, 2004. 39th IAS Annual Meeting, Seattle, WA, USA, 3-7 October 2004; Volume 41, pp. 1548-1556. [CrossRef] 